\title{
Pratiques du travail au forfait. Métiers, techniques et sous-traitance dans une perspective euro-asiatique, $\mathrm{XVIII}{ }^{\mathrm{e}}-\mathrm{XXI}{ }^{\mathrm{e}}$ siècles. Une introduction
}

\author{
Manuela Martini*, Liliane Hilaire-PÉrez**, Giorgio Riello***
}

$\mathrm{L}$ oin d'être une forme d'organisation de la production et du travail inhérente la crise qui secoue les économies occidentales depuis les années 1970, la sous-traitance est une pratique de long terme inscrite dans une pluralité de contextes productifs. Ce numéro spécial a pour objectif d'explorer la sous-traitance dans les secteurs économiques particulièrement concernés, certains ayant fait l'objet de l'observation attentive des contemporains, économistes,

* Manuela MARTini est professeure d'histoire contemporaine à l'Université Lumière Lyon 2 et membre du Laboratoire de recherche historique Rhône-Alpes (LARHRA-UMR 5190). Parmi ses publications récentes figurent Bâtiment en famille. Migrations et petite entreprise en banlieue parisienne au XX ${ }^{e}$ siècle (Paris, CNRS Éditions, 2016), et l'édition de What is work? Gender at the Crossroads of Home, Family and Business from the Early Modern Era to the Present (New York/Oxford, Berghahn, 2018), avec Raffaella Sarti et Anna Bellavitis. Adresse : LARHRA-MSH Lyon Saint-Étienne, 14 avenue Berthelot, 69007 Lyon (manuela.martini@univ-lyon2.fr).

* Liliane Hilaire-Pérez est professeure d'histoire moderne à l'Université de Paris, membre du laboratoire Identités, Cultures, Territoires (ICT-EA 337) et directrice d'études à l'EHESS, membre du Centre Alexandre Koyré. Elle a publié son mémoire d'habilitation sous le titre La pièce et le geste. Artisans, marchands et savoirs techniques à Londres au XVIII ${ }^{e}$ siècle (Paris, Albin Michel, L'Évolution de l'Humanité; 2013). Elle est directrice de la revue ARTEFACT. Techniques, histoire et sciences humaines. Adresse: UFR GHES, Université de Paris, 5, rue Thomas Mann 75205 CEDEX 13 Paris. Case courrier 7001 (liliane.perez@wanadoo.fr).

*** Giorgio Riello est en poste sur la chaire d'Histoire globale de l'époque moderne de l'Institut universitaire européen, Florence, en disponibilité de l'université de Warwick, Royaume-Uni. Il est l'auteur de plusieurs livres dans le domaine de l'histoire globale, parmi lesquels Cotton: The Fabric that Made the Modern World (Cambridge, Cambridge University Press, 2013), lauréat du prix de la World History Association en 2014 et Luxury: A Rich History, avec Peter McNeil (Oxford, Oxford University Press, 2016). Adresse: Department of History and Civilization, European University Institute, Via Bolognese 156, 50139 Florence, Italy (giorgio.riello@eui.eu). 
juristes, administrateurs et acteurs économiques dans leur ensemble. Nous considérons donc le rôle historique des formes de production alternatives à la concentration usinière, en nous penchant sur les relations de sous-traitance comme faisant partie intégrante de l'organisation artisanale et industrielle.

Depuis les années 1980, le thème de la spécialisation flexible est au centre de l'attention des historiens sous la bannière des « alternatives historiques à la production de masse ${ }^{1} »$. La sous-traitance et d'autres formes flexibles d'organisation de la production sont des sujets maintenant bien connus en histoire économique et en sciences sociales ${ }^{2}$. Dans la littérature récente, ils ont été étudiés soit comme des formes idéales de flexibilité industrielle, soit inversement, comme relevant de l'exploitation des travailleurs. En période de crise et de reconfiguration industrielle comme dans les années 1970, la sous-traitance a été saluée comme la solution à la rigidité des systèmes de production tayloristes et fordistes. Dans le même temps, cependant, la sous-traitance a été considérée comme fortement liée à des formes d'exploitation de la maind'œuvre, qu'il s'agisse du travail de force dans les métropoles du XIX ${ }^{\mathrm{e}}$ siècle ou dans les ateliers textiles actuels, en particulier en Asie.

Ce numéro spécial a pour but de proposer une nouvelle approche de cette forme majeure et durable d'organisation de la production et du travail. Premièrement, nous insistons sur la nécessité de comparer dans le temps et dans l'espace les différentes expériences de sous-traitance et d'autres formes de «fragmentation de la production» tels que le travail domestique, le travail à la pièce, les contrats informels entre entreprises, les entreprises familiales et les travailleurs indépendants. Malgré le large éventail de formes que prend la sous-traitance, nous souhaitons mettre en évidence les caractéristiques communes de ce mode d'organisation de la production. Deuxièmement, nous voulons souligner la nécessité de mieux comprendre les logiques internes des relations de sous-traitance et de fonctionnement des ateliers, et en particulier le rôle joué par les sous-traitants. À travers des études de cas empiriques, on observe une gamme assez complexe d'interrelations dans la séquence que recouvre la sous-traitance. Souvent victimisés en tant que main-d'œuvre exploitée, les derniers maillons de la chaîne ont souvent exprimé leur capacité d'agir. La logique interne de la sous-traitance montre que la distinction entre employeur et employé est fluide selon les conditions du marché, du capital et de l'entreprise. Les articles qui forment ce numéro spécial contribuent donc au débat sur le statut juridique des sous-traitants, des donneurs d'ordre et des

1 Sabel, Zeitlin, 1997.

2 Salais, Storper, 1993. 
chaînes de sous-traitance ${ }^{3}$. Enfin, nous soulignons l'importance de contextualiser la sous-traitance dans le rapport marchand aux consommateurs et aux distributeurs. L'évolution de la consommation (variété des produits demandés et, plus récemment, personnalisation) a souvent entraîné la pratique de la sous-traitance comme un moyen de répondre rapidement et efficacement à l'instabilité des choix des consommateurs ${ }^{4}$.

\section{GENÈSE D'UN CONCEPT}

La sous-traitance est aussi difficile à définir qu’à cerner historiquement. Il s'agit des services fournis par un «sous-traitant» à une «entreprise acheteuse » ou à un «donneur d'ordre » pour la production de pièces, composants ou assemblages destinés à être incorporés dans un produit que le donneur d'ordre va commercialiser 5 . Les relations de sous-traitance étant omniprésentes dans les systèmes manufacturiers occidentaux et non-occidentaux, du moins depuis le Moyen Âge européen, si ce n'est plus tôt, leur étude s'est appuyée à la fois sur une diversité d'analyses historiques et sur les approches théoriques de plusieurs disciplines en sciences sociales. Parmi elles, il faut citer le domaine de l'économie industrielle et en particulier l'attention accordée depuis les années 1960 au rôle joué par les institutions dans le développement économique. L'histoire des entreprises a également joué un rôle important dans la compréhension de la sous-traitance, notamment à travers l'analyse des différentes typologies d'organisation de la production. De même, les relations de sous-traitance ont été façonnées par des réglementations des droits des travailleurs, des sous-traitants et des donneurs d'ordre, principalement dans le domaine de la législation du commerce et du travail. Au cours des dernières décennies, la sociologie et l'anthropologie ont ouvert de nouvelles pistes dans l'étude de la sous-traitance en examinant le quotidien des travailleurs, leur appartenance sociale et leur expérience du travail ${ }^{6}$.

En raison peut-être de sa polyvalence, la sous-traitance a été traitée de manière différenciée dans les débats économiques, sociétaux et culturels. Il convient de souligner trois contextes d'analyse. Premièrement, la discipline économique s'est penchée sur la question des frontières de l'entreprise, des

3 Hilaire-PÉrez, 2013; Martini, 2016.

4 Riello, 2008 ; Perry, 1999.

5 Martini, 2015b. Pour une définition juridique voir Valentin, 1979; Braudo, https ://www .dictionnaire-juridique.com/definition/sous-traitance.phpand consulté le 4/01/2019.

6 Perraudin, Thèvenot, Tinel, Valentin, 2006. 
limites de l'intégration verticale et de ce qui constitue les activités centrales d'une entreprise. En particulier, les travaux de Ronald H. Coase dans les années 1930 et ceux d'Oliver E. Williamson dans les années 1970 et 1980 sur l'économie des coûts de transaction ont fourni une large conceptualisation de la diversité des entreprises déterminée par des configurations particulières des coûts encourus pour rechercher, négocier et surveiller les transactions. ${ }^{7}$ Ce type de recherche ne s'intéressait pas spécifiquement à l'évaluation de la nature ou des avantages de la sous-traitance ; en revanche, il tentait de décrire la sous-traitance comme offrant un moyen d'internaliser les transactions, réduisant ainsi le coût qu'un agent économique aurait autrement dû supporter en s'adressant au marché. La recherche sur les coûts de transaction a eu pour corollaire une compréhension non-économique à la fois des transactions marchandes menées par des entreprises et des relations contractuelles comme la sous-traitance. Il a été noté, par exemple, que dans les sociétés où la confiance fait partie d'un système de valeurs socialement partagées et où les violations sont désapprouvées par la société, la sous-traitance fonctionne bien. Un exemple est le Japon, où les sous-traitants ont une tendance beaucoup plus faible aux litiges qu'aux États-Unis, par exemple 8 .

Comme nous l'avons déjà mentionné, la sous-traitance est apparue comme un concept à part entière (plutôt que comme une exception à l'internalisation de fonctions au sein des entreprises) au cours des années 1970 et 1980. Deux changements méritent d'être mentionnés : d'abord une réévaluation de la petite entreprise et ensuite, la recherche de modèles de production industrielle plus flexibles. Lorsqu'en 1973 Ernst Schumacher a publié pour la première fois son livre très remarqué Small is Beautiful, le paradigme existant était d'inspiration chandlérienne et faisait la promotion des atouts des grandes entreprises verticalement intégrées. Schumacher a ouvert une nouvelle voie de recherche sur les petites entreprises, affirmant que celles-ci ne doivent pas être considérées comme des entreprises qui ont échoué dans leur recherche d'expansion et de croissance ou comme les traces de structures productives « traditionnelles » et plutôt arriérées. En revanche, selon lui, les petites entreprises pouvaient être à la pointe de l'innovation (un concept aujourd'hui entièrement intégré dans le concept de «start-up ») et flexibles dans leur organisation de la production ${ }^{9}$.

Cette réévaluation des petites entreprises était aussi une critique du modèle industriel de production de masse, un processus de fabrication de produits standard réalisés par des machines hautement spécialisées et des travailleurs

7 Coase, 1937 ; Williamson, 1979 ; Williamson, 1985. Voir aussi LANGlois, 2004.

8 Williamson, 1986 ; Thoburn, Takashima, 1992.

9 Cette fameuse formule est due à SCHUMACHER, 1973. 
semi-qualifiés qui avaient été omniprésents dans l'organisation de la production depuis au moins la deuxième révolution industrielle. L'enjeu de la recherche à la fin des années 1970 était d'aller au-delà de la production de masse, ce qui imposait une redéfinition et une analyse en termes politiques des choix techniques et institutionnels nécessaires à la production de biens pour des marchés de plus en plus segmentés mais aussi pour des consommateurs toujours plus exigeants. L'idée de lier la crise à la rigidité du système de production de masse est courante aujourd'hui, mais au début des années 1970, elle n'avait sa place ni dans la théorie ni dans les travaux empiriques de l'histoire des entreprises. Il a fallu un économiste peu orthodoxe comme Michael J. Piore et des politologues ayant une connaissance approfondie des différents paysages industriels européens comme Charles Sabel et Jonathan Zeitlin pour concevoir un modèle de spécialisation flexible comme alternative au fordisme.

Cette recherche originale a donné lieu à la publication par Michael Piore et Charles Sabel de The Second Industrial Divide (1984), à l'article essentiel de Sabel et Jonathan Zeitlin en 1985 « Alternatives to mass production » et à leur recueil collectif Alternatives to Mass Production (1997 $)^{10}$. Contrairement au modèle de production de masse de la grande entreprise verticalement intégrée, celui de la spécialisation flexible a suscité de nombreuses études sur les zones industrielles et les systèmes de production localisés. Suzanne Berger et Harry Katz, Giacomo Becattini, Michele Salvati, Sebastiano Brusco, Robert Boyer et Jean-Jacques Silvestre, ainsi que des sociologues des districts industriels et des systèmes de production locaux tels que Vittorio Capecchi, Carlo Trigilia et Arnaldo Bagnasco ont joué un rôle important dans ce débat des années $1990^{11}$.

Ce qui a marqué les débats sur la spécialisation flexible et les alternatives à la production de masse est son caractère historique. Au début des années 1980, on a reconnu que le processus de « modernisation » économique - plutôt que de simple «industrialisation»- recouvrait une grande variété d'évolutions et ne pouvait plus reposer sur des hypothèses positivistes de croissance ou de développement unilatéral. Il en a résulté une réévaluation de la pluralité des voies de développement économique et une prise en compte approfondie des systèmes productifs urbains, en particulier aux XVIII ${ }^{\mathrm{e}}$ et XIX ${ }^{\mathrm{e}}$ siècles. Il convient de mentionner ici le travail de Maxine Berg et Pat Hudson sur la production manufacturée ou la compréhension historique des districts industriels

\footnotetext{
10 Piore, Sabel, 1984 ; Sabel, Zeitlin, 1985 ; Sabel, Zeitlin, 1997 ; Fuà, 1985.

11 Des remarques sur l'origine de ce champ de recherche dans MARTINI, 2015a.
} 
comme par exemple dans un corpus d'études aujourd'hui important en Italie ou en France ${ }^{12}$.

Aujourd'hui, la sous-traitance n'est plus considérée comme liée à la recherche d'alternatives industrielles à la production de masse, ni même à la recherche de flexibilité. Depuis les années 1980, la sous-traitance à l'échelle mondiale a attiré l'attention d'organisations internationales comme l'OCDE (Organisation de coopération et de développement économiques) et l'ONUDI (Organisation des Nations Unies pour le développement industriel). D'autres perspectives sur ces politiques sont apparues dans le contexte plus large des critiques de la mondialisation ${ }^{13}$. On a interprété la sous-traitance au niveau mondial comme une forme de division capitaliste du travail et d'exploitation par les pays industrialisés des pays du Sud. Des thèmes connexes tels que les ateliers intensifs, le travail domestique et les questions de genre dans certains métiers ont été transposés à une échelle globale ${ }^{14}$. Plus récemment, cette approche critique a donné lieu à des plaidoyers en faveur d'une sous-traitance mondialisée respectueuse des individus et de l'environnement ${ }^{15}$.

Les réseaux mondiaux intersectoriels de production et de construction de la valeur des biens révèlent des formes complexes et non-linéaires de sous-traitance. C'est le plus évident dans la production de biens de consommation, par exemple les chaussures de sport ou l'industrie de l'habillement en Chine. Gilles Guiheux montre dans ce numéro spécial que c'est le cas des grandes entreprises produisant pour des marchés occidentaux dont les clients sont attentifs à l'ensemble du cycle de production ou dans le cas de chaînes de petites et moyennes entreprises produisant des vêtements bas de gamme en Chine urbaine contemporaine pour le marché intérieur ou, parfois, pour des acheteurs étrangers ${ }^{16}$.

La sous-traitance à l'ère de la mondialisation comprend des formes d'externalisation, de délocalisation, de partenariat et de travail dispersé. En effet, aujourd'hui, la sous-traitance est étiquetée différemment selon l'acteur économique sur lequel on met l'accent, le donneur d'ordre ou le sous-traitant, et selon

12 Berg, Hudson, Sonenscher, 1983; Berg, 1991; Berg, 1985. La littérature sur les districts industriels est énorme dans le cas de l'Italie et deviant un sujet d'analyse aussi pour la Grande-Bretagne et la France. Par exemple : Popp, 2001 et Wilson, Popp, 2003, notamment l'introduction. Pour la France, voir Daumas, 2007; ECK, Lescure, 2002; GANNE, 1989 et 1995.

13 Martini, 2015b.

14 Boris, 1994; TOFFANin, 2016.

15 Robinson, 2008 (voir en particulier "Global economy and Latin America II : industrial subcontracting, transnational services, tourism, and the export of labor", p. 102-166).

16 Riello, 2008. 
le type de relation qui est soulignée. Les services sont la nouvelle frontière de la sous-traitance grâce aux nouvelles technologies et en particulier à Internet. Dans tout pays européen, le service à la clientèle peut nécessiter la présence physique d'ingénieurs sur sites, mais l'assistance technique et la communication peuvent être sous-traitées à des pays asiatiques. Il en va de même pour la nouvelle «gig economy» où la relation entre la libre entreprise et la dépendance est souvent difficile à évaluer. Les plates-formes en ligne permettent la participation au marché du travail sur une base flexible tout en remettant en question bon nombre des hypothèses concernant les droits des travailleurs, l'assurance maladie et les avantages sociaux tels que les congés rémunérés qui avaient été obtenus à l'époque de la production industrielle.

\section{POURQUOI SOUS-TRAITER?}

Les auteurs des articles de ce numéro ne considèrent pas la sous-traitance comme une aberration du système productif ou un simple moyen de résoudre les problèmes du marché, mais comme un élément intrinsèque des formes de production ou d'exploitation commerciale, que ce soit en Chine, en Malaisie ou en Europe, du début des temps modernes à nos jours. Selon les secteurs et les types de produits, la sous-traitance répond à des exigences différentes: dans le cas des travaux publics (Anne Conchon), de la construction (Manuela Martini), des chantiers navals (David Plouviez) ou de la production de produits agricoles commercialisables (gambier, huile de palme) qui constituent des matières premières industrielles (Éric Guerassimoff), elle permet de résoudre des problèmes logistiques et de maîtriser les coûts. En ce qui concerne la production et la distribution de biens de consommation (tissus, vêtements, jouets, chaussures), la sous-traitance garantit une certaine flexibilité dans des contextes de demande changeante (Gilles Guiheux) mais aussi une coordination plus souple de la production industrielle et de la distribution (Carles Brasó). Enfin, pour les produits de luxe, elle permet d'optimiser la coordination de métiers de haute qualification comme dans l'horlogerie, qu'ils aient été amenés à collaborer dans le passé (Liliane Hilaire-Pérez et Helen Clifford) ou aujourd'hui dans l'horlogerie suisse contemporaine ${ }^{17}$.

Chaque article de ce volume illustre la complexité d'identifier les mécanismes par des relations de sous-traitance. De la Grande-Bretagne et de la France du XVIII e siècle à la Malaisie du XIX ${ }^{\mathrm{e}}$ siècle, en passant par la Chine contemporaine, l'étude de la sous-traitance exige le croisement de sources 
qui comprennent les procédures judiciaires (telles les archives cruciales issues des procès de la chancellerie, à Londres), les bilans de faillite, les registres des litiges, les référés et jugements des tribunaux du travail, les rapports des commissaires et intendants, etc. Il s'agit de «lire entre les lignes», mais comme le fait remarquer David Plouviez, de nombreux documents nécessaires pour reconstituer une image complète de la sous-traitance, comme dans le cas de la marine française, n'existent plus. L'analyse des contrats privés signés entre l'État et les entrepreneurs qui approvisionnent les marchés de la construction navale est plus fructueuse, mais également chronophage. Ceci est le cas également pour l'étude des adjudications entre l'appel d'offres de l'État et le cahier des charges proposée par Anne Conchon. Les archives juridiques, les actes issus de procédures d'arbitrage du travail, la législation et les référés (des Prud'hommes notamment en France) tels qu'analysés par Manuela Martini sont une autre piste d'enquête, tout comme les sources primaires utilisées par Éric Guerassimoff qui comprennent les archives malaisiennes et européennes complétées par des informations tirées de l'épigraphie chinoise et des sources écrites, des témoignages oraux de descendants et des informations inédites à partir de témoignages des missionnaires français. Également révélatrices sont les statistiques industrielles ainsi que les rapports d'activité des grandes entreprises utilisés par Carles Brasó.

Ce qui distingue ce numéro tient donc d'abord à la diversité des sources. Mais les textes révèlent aussi l'impossibilité d'apporter une explication mono-causale à la sous-traitance. La question en apparence simple « pourquoi la sous-traitance existe-t-elle ?» trouve des réponses variées. Liliane HilairePérez et Helen Clifford, par exemple, considèrent la sous-traitance dans les économies métropolitaines du XVIII ${ }^{\mathrm{e}}$ siècle à Londres et Paris comme un véritable moteur de la croissance économique. L'analyse d'Anne Conchon des besoins productifs et des infractions dans les travaux publics en France sous l'Ancien Régime permet de voir dans la sous-traitance un moyen incontournable pour faire face aux pénuries de main-d'œuvre et aux problèmes techniques. De même, David Plouviez souligne la nécessité de recourir à la sous-traitance pour surmonter les problèmes logistiques. La sous-traitance a permis de gérer la complexité du monde pré-industriel. La clé est l'accès à des qualifications spécifiques rassemblant souvent différentes combinaisons de compétences dans des processus de production complexes. C'est le cas de l'horloger londonien James Cox (1723-1800) qui dit avoir eu recours à plus de 600 travailleurs à domicile, tandis que les orfèvres Parker et Wakelin ont employé 75 entrepreneurs de 1766 à 1770, dont des artisans à Birmingham et Sheffield, les deux 
centres émergents dans la métallurgie au XVIII e siècle en Angleterre. Anne Conchon se demande si la sous-traitance dépend de la nature des tâches, de leur difficulté technique, de la taille des sites ou du degré d'urgence des travaux à réaliser. Dans un monde où la plupart des artisans n'avaient pas la capacité de mobiliser des compétences techniques et de la main-d'œuvre qualifiée, et ne disposaient pas du capital nécessaire pour mener à bien des tâches complexes, la sous-traitance représentait une forme de «désintégration», notamment de la production urbaine. Dans une continuité frappante, Manuela Martini souligne l'importance dans l'industrie française de la construction du XIX ${ }^{\mathrm{e}}$ siècle de sous-traitants qualifiés capables de suivre les travailleurs dans un contexte de pénurie de main-d'œuvre sur le marché du travail ou de manque de spécialités ou d'artisans. Alors que la théorie de l'entreprise souligne à quel point l'internalisation est essentielle à la réalisation d'économies d'échelle grâce à des machines spécialisées, la sous-traitance met plutôt l'accent sur le rôle des compétences. Pourtant, comme le montre David Plouviez, l'accès aux habiletés ne se limite pas aux économies urbaines ou à la fabrication de biens de consommation. Dans les années 1630, la marine française en développement a fait appel à des charpentiers néerlandais pour la construction de certaines parties de ses navires, ce qui a donné naissance à des navires avec des lignes plus basses, réduisant la taille des châteaux arrière et augmentant l'artillerie.

Un deuxième sujet important qui ressort de toutes les contributions est la relation entre la sous-traitance et la division du travail. Adam Smith voit dans ce dernier point une source de différenciation technique et d'amélioration des compétences. La division du travail était un sujet de grande préoccupation, en particulier pour les corporations qui dominaient les métiers traditionnels dans l'Europe préindustrielle. Leur système de formation basé sur l'apprentissage comportait l'idée d'une unité des tâches dans la production de biens différents (chaussures, orfèvrerie, etc.) mais il était difficile de conceptualiser le travail de différentes manières, notamment en termes de tâches. Il y avait plus de liberté en dehors du système corporatif. David Plouviez, par exemple, observe quà la fin du XVIII ${ }^{\mathrm{e}}$ siècle, la marine française construisait depuis longtemps ses navires en vingt-quatre parties correspondant à un ensemble de tâches prédéfinies. Il s'agissait également d'un modèle utilisé dans la production de biens complexes, comme par exemple des carrosses ou des horloges composées de pièces spécialisées. Les livres de comptes de l'association entre Benjamin Gray et François Justin Vulliamy dans la seconde moitié du XVIII siècle montrent que la moitié des quarante-trois travailleurs extérieurs se trouvaient dans les quartiers de Soho et Saint-Giles-in-the-Fields à Londres. À propos des entreprises de Gray et Vulliamy, Liliane Hilaire-Pérez et Helen Clifford observent 
qu'il fallait créer une nouvelle épistémologie basée sur la compréhension du travail comme principes opératoires, à travers la comptabilité, pour la bonne gestion de l'entreprise. Ces auteures montrent que les métiers furent remodelés en fonction de la spécialisation des produits - un phénomène déjà visible dans la prolifération de nouvelles corporations à Londres et à Paris dans la seconde moitié du XVII ${ }^{e}$ siècle. Cependant, cela signifiait la disparition des identités artisanales traditionnelles : « Les artisans sous-traitants ne travaillaient plus en tant qu'artisans même s'ils n'agissaient pas encore en tant que mécaniciens ». Cette nouvelle épistémologie exigeait aussi une nouvelle terminologie que nous voyons se développer du XVIII ${ }^{\mathrm{e}}$ au XX ${ }^{\mathrm{e}}$ siècle. Cela inclut les expressions, comme dans les travaux publics décrits par Anne Conchon telles qu'«adjudication au rabais», «marché à prix faits » ou «marché à l'entreprise » qui font référence de façon récurrente à la sous-traitance par opposition au travail « par économie » ou «journée du roi ». Un siècle plus tard, dans l'industrie de la construction étudiée par Manuela Martini, on trouve des expressions similaires comme «à la tâche », «à la pièce », « au forfait», pour indiquer les formes de rémunération les plus souvent utilisées par les différents corps de métier qui se côtoient dans la fabrication d'un bâtiment, et contrairement aux travaux de terrassement et de fouille généralement payés à la journée.

Troisièmement, la multiplicité des définitions des modes de rémunération du travail va de pair avec la pluralité des types de sous-traitants. Les frontières entre les travailleurs autonomes et les entrepreneurs sont floues. Les types d'intermédiaires vont des artisans travaillant avec un membre de leur famille aux hommes d'affaires qui gèrent d'énormes fonds, des sous-traitants travaillant pour une seule grande entreprise et dans un état semi-subordonné à ceux qui diversifient leurs activités entre différentes entreprises. En Europe, le caractère entrepreneurial de l'activité de sous-traitance n'est pas clairement défini entre la fin du XIX et le début du XX ${ }^{\mathrm{e}}$ siècle : le degré d'autonomie du « tâcheron» par rapport au donneur d'ordre est interrogé par les théoriciens et les membres des tribunaux et conseils arbitraux du travail. La distinction n'est pas facile à définir, comme le montre Manuela Martini pour les travailleurs de la construction ou Éric Guerassimoff pour les intermédiaires chinois en Malaisie, surtout lorsque le sous-traitant est un «entrepreneur de main-d'œuvre », un profil spécifique de sous-traitant qui apparaît non seulement dans les traités théoriques mais qui aussi est le sujet bien réel de litiges judiciaires et donc de débats juridiques. Dans une perspective macro-industrielle, Carles Brasó souligne que la sous-traitance reste inhérente aussi aux organisations usinières. À partir du cas de la production lainière en Chine entre la fin du XIXe siècle et la Deuxième Guerre mondiale, il souligne comment les petites et moyennes entreprises ont continué à cohabiter avec les grands groupes et comment ces 
derniers sous-traitaient les phases finales de la production (les finitions, mais aussi la teinture et la couture) et de la distribution à des petits ateliers.

$\mathrm{Au}$ cœur de la conceptualisation de la sous-traitance se trouve aussi la nouvelle importance accordée à la consommation. D'une part, la sous-traitance a été vue comme une forme d'organisation de la production, d'autre part, on a mis en avant le concept d'une «économie du produit». La sous-traitance a permis non seulement l'acquisition de compétences mais aussi, par la recombinaison constante, la création d'une variété de produits. Ce n'est pas un hasard si la sous-traitance a été particulièrement importante dans la production des carrosses, montres et horloges, instruments, meubles, chaussures et autres biens de consommation. C'est le domaine du "populuxe» du XVIII e siècle - qui combine l'attrait du luxe mais recouvre des produits moins chers - comme c'est le cas aujourd'hui de la production occidentale de produits de marque (et de luxe) en Chine et dans d'autres pays asiatiques (Gilles Guiheux).

La sous-traitance a eu un impact non seulement sur les produits et leur prix, mais aussi sur l'économie artisanale. Déjà en 1726, Daniel Defoe faisait la distinction entre « ceux qui ne fabriquent pas vraiment les biens qu'ils vendent», « ceux qui fabriquent seulement des biens destinés à d'autres qui les vendent » et « ceux qui font les biens qu'ils vendent alors qu'ils tiennent boutique ».

La sous-traitance n'existe cependant pas seulement dans un « régime de pratiques ». Depuis le Moyen-Âge, elle fait l'objet de débats et d'interventions sur le plan législatif. Dans l'impossibilité de contrôler cet ensemble de pratiques et leurs évolutions, les régimes juridiques passés nous ont laissé de nombreuses traces de conflits. On peut donc considérer la sous-traitance à la lumière de l'économie des contrats. Dans les travaux publics français du XVIII e siècle, par exemple, la sous-traitance était formellement interdite ou strictement contrôlée pour éviter toute confusion quant à la responsabilité juridique et tout risque d'une augmentation des coûts. Dans la pratique, cependant, comme Anne Conchon l'observe, la sous-traitance est apparue comme une réponse rationnelle au caractère restrictif des concours de marchés publics de travaux publics, dans lesquels seules des entreprises agréées disposant d'une bonne capacité financière étaient en mesure de concourir. Au milieu du XIX ${ }^{\mathrm{e}}$ siècle, l'interdiction du «marchandage » proclamée par la Seconde République en France se traduit en effet, notamment dans le secteur de la construction, par la répression de ces abus. Rien à voir avec leur abolition. Malgré la résurgence, notamment au début du XX ${ }^{\mathrm{e}}$ siècle, de l'action syndicale contre toute forme de «marchandage », la législation durant tout le siècle réitère la position de la jurisprudence de la fin du XIX ${ }^{\mathrm{e}}$ siècle. Comme Manuela Martini le démontre, ce n'est qu'à la fin des années 1930 que la législation apparaît comme le point crucial du statut légal de sous-traitant: si le sous-traitant n'est pas inscrit dans 
les registres commerciaux, et n'est donc pas un entrepreneur légal, la responsabilité de l'entrepreneur principal envers les travailleurs du sous-traitant ne sera pas dégagée.

Un autre trait spécifique est l'importance des questions comptables liées à la sous-traitance. En poursuivant la démonstration de Liliane Hilaire-Pérez et Helen Clifford en faveur de la création d'une nouvelle épistémologie de l'artisanat, on peut considérer que la sous-traitance est également caractérisée par la production d'un nouveau «système d'information » fondé sur une gestion financière, des plans et des devis de construction détaillés afin que les entrepreneurs disposent des informations suffisantes pour pouvoir exécuter leurs contrats.

Dans un tout autre contexte, Éric Guerassimoff observe que le système des intermédiaires chinois appelés Kangchu en Malaisie entre le XVIII e et le début du XX ${ }^{\mathrm{e}}$ siècle mobilise les compétences, y compris les capacités de crédit et de comptabilité nécessaires à la création d'entreprises assez complexes. L'utilisation de systèmes d'information sophistiqués caractérise aujourd'hui la sous-traitance. Les chaînes de production mondiales et de construction de la valeur des produits telles que définies par Gary Gereffi relient différents marchés internationaux et suivent la logique d'une production distendue. Comme l'observe Gilles Guiheux, au cours des dernières décennies, la mondialisation des chaînes de valeur initiée par les grandes enseignes du Nord a été l'un des moteurs du développement de l'industrie du prêt-à-porter en Chine. Le système productif se caractérise par l'interdépendance de petites unités au sein d'une zone géographique donnée similaire à celle d'un district marshallien. Pourtant, la logique de la sous-traitance mondiale d'aujourd'hui répond moins à une question de production qu'à la capacité à satisfaire rapidement et à moindre coût les marchés de consommation mondiaux.

En guise de conclusion, nous soulignerons l'importance de l'étude de la sous-traitance pour une histoire sociale du travail et de l'entreprise. Les recherches jusqu’à présent se sont concentrées sur la pluralité des mondes de production, sur les interactions incessantes entre la production et la consommation en prenant en compte la variabilité de la demande, y compris en plein $\mathrm{XX}^{\mathrm{e}}$ siècle à l'ère de la production de masse, ainsi que sur la dimension globale du phénomène d'allongement des circuits, soit sur la malléabilité de cette forme de production, intégrée à de multiples stratégies, dont celles du commerce mondialisé. Si sous l'Ancien Régime, comme l'a montré Jean-Yves Grenier, « l'absence de réalisation totale du travail sur le marché résulte donc du fait que le rapport de production préindustriel se caractérise moins par l'achat de la force de travail que par le contrôle extérieur du processus de production 
opéré par celui de l'échange et de la commercialisation ${ }^{18}$ », une telle organisation de la production a traversé les époques et trouvé de multiples expressions contemporaines. Ce sont là des acquis essentiels de la recherche. Mais une autre thématique se profile. Les enquêtes sur les liens entre la sous-traitance et le travail domestique et féminin d'une part, les travaux sur la densification des micro-ateliers en milieu urbain depuis l'époque moderne, d'autre part, suggèrent que sur le plan social, ce mode de production va de pair avec l'invisibilisation de ses acteurs. Il est sûr que la mixité des statuts socio-économique interdit de victimiser les sous-traitants qui peuvent très bien être donneurs d'ordre à leur tour. Néanmoins, sur un plan structurel, une telle organisation rend le processus de production moins lisible et tend à reléguer les acteurs dans un anonymat dont ils sont, au contraire, relativement préservés en corporations ou dans le cadre usinier, même dans la petite fabrique comme l'atteste la tradition des photographies du personnel. Les sous-traitants appartiennent au monde des réseaux, des intermédiaires, des marchés de production ${ }^{19}$. Leur invisibilisation a même pu constituer une stratégie politique comme le montre Chandra Mukerji pour le Canal du Midi, un chantier sous Louis XIV marqué par la reprise en main royale du territoire en passant contrat avec des sous-traitants modestes, en concurrence d'entrepreneurs réputés, soit une politique visant l'effacement du travail créatif ${ }^{20}$. Alors que de nos jours, s'accentue la désindustrialisation et que le monde ouvrier est rendu invisible en société, l'histoire de la sous-traitance permet d'analyser sur le long terme les multiples formes de dilution du travail productif. La vigueur de ce champ de recherche est à mesure des enjeux sociétaux posés par les tranformations du secteur secondaire.

\section{LISTE DES RÉFÉRENCES}

Berg (Maxine), 1985, The Age of Manufactures: Industry, Innovation and Work in Britain, 1700-1820, Londres, Fontana.

BERg (M.) (dir.), 1991, Markets and Manufacture in Early Industrial Europe, Londres, Routlegde.

Berg (Maxine), Hudson (Pat), Sonenscher (Michael) (dir.), 1983, Manufacture in

Town and Country Before the Factory, Cambridge, Cambridge University Press.

$\begin{array}{ll}18 & \text { Grenier, 1996, p. } 21 . \\ 19 & \text { White, 2002. } \\ 20 & \text { Mukerji, } 2009 .\end{array}$


Boris (Eileen), 1994, Home to Work. Motherhood and the Politics of Industrial Homework in the United States, Cambridge, Cambridge University Press.

Braudo (Serge), Dictionnaire du droit privé, 'Sous-traitance', https ://www.dictionnaire -juridique.com/definition/sous-traitance.php [consulté le 4 janvier 2019].

CoAse (Ronald H.), 1937, « The Nature of the Firm », Economica, 4, p. 386-405.

DAumas (Jean-Claude), 2001, «Districts industriels: du concept à l'histoire. Les termes du débat », Revue économique, 58-1, p. 131-152.

Donzé (Pierre-Yves), 2005, Formation professionnelle et développement industriel dans le district de Porrentruy aux $19^{e}$ et $20^{e}$ siècles, Neuchâtel, Alphil.

Eck (Jean-François), Lescure (Michel) (dir.), 2002, Villes et districts industriels en Europe occidentale, XVII ${ }^{e_{-}} X X^{e}$ siècles, Tours, Presses Universitaires François-Rabelais.

FUÀ (Giorgio), 1985, « Les voies diverses du développement en Europe », Annales ESC, 3, p. 579-603.

GANNE (Bernard), 1989 «Pme et districts industriels : quelques réflexions critiques à propos du "modèle italien" », Revue internationale pme, 2-3, p. 276-277.

GANNE (B.), 1995 «France : behind small and medium size enterprises lies the State», dans Bagnasco (Arnaldo), SABEL (Charles) (dir.), Small firms in Europe, Londres, Pinter, p. 115-133.

Grenier (Jean-Yves), 1996, L'économie d'Ancien Régime. Un monde de l'échange et de l'incertitude, Paris, Albin Michel.

Hilaire-PÉrez (Liliane), 2013, La pièce et le geste: artisans, marchands et savoir technique à Londres au XVIII e siècle, Paris, Albin Michel.

LANGlois (Richard N.), 2004, "Chandler in a Larger Frame: Markets, Transaction Costs, and Organizational Form in History," Enterprise \& Society, 5, p. 355-375.

Martini (Manuela), 2015a, «Second Industrial Divide (The)», Encyclopedia of Economics and Society, coord. Frederick WHERRY General Editor, SAGE Reference p. 1457-1459.

Martini (M.), 2015b, «Subcontracting», Encyclopedia of Economics and Society, coord. Frederick WHERRY General Editor, SAGE Reference, p. 1540-1542.

Martini (M.), 2016, Bâtiment en famille. Migrations et petite entreprise en banlieue parisienne au $X X^{e}$ siècle, Paris, CNRS Éditions.

MukERJi (Chandra), 2009, Impossible Engineering: Technology and Territoriality on the Canal du Midi, Princeton, Princeton University Press.

PERry (Martin), 1999, Small Firms and Network Economies, Londres et New York, Routledge.

Piore (Michael J.), SABEL (Charles F.), 1984, The Second Industrial Divide: Possibilities for Prosperity, New York, Basic Books.

Popp (Andrew), 2001, Business Structure, Business Culture, and the Industrial District: the Potteries, c. 1850-1914, Aldershot, Ashgate. 
RiELLO (Giorgio), 2008, «Strategies and boundaries : subcontracting and the London trades in the long eighteenth century », Enterprise \& Society, 9-2, p. 43-80.

Robinson (William I.), 2008, Latin America and Global Capitalism. A Critical Globalisation Perspective. Baltimore, The Johns Hopkins University Press.

SABEL (Charles F.), Zeitlin (Jonathan) (dir.), 1985, « Historical Alternatives to Mass Production », Past \& Present, 108, 133-176.

SAbel (Ch. F.), Zeitlin (J.) (dir.), 1997, World of Possibilities: Flexibility and Mass Production in Western Industrialization, New York, Cambridge University Press.

SAlais (Robert), Storper (Michael) (dir.), 1993, Les mondes de production. Enquête sur l'identité économique de la France, Paris, Éd. de l'EHESS.

Schumacher (Ernst F.), 1973, Small is Beautiful: a Study of Economics as if People Mattered, Londres, Blond \& Briggs.

Thoburn (John), Takashima (Makoto), 1992, Industrial Subcontracting in the UK and Japan, Aldershot, Avebury.

Toffanin (Tania), 2016, Fabbriche invisibili. Storie di donne, lavoranti a domicilio, Verona, Ombre Corte.

VALentin (Georges), 1979, Les contrats de sous-traitance, Paris, Librairies Techniques.

White (Harrison C.), 2001, Markets from Networks. Socioeconomic Models of Production, Princeton-Oxford, Princeton University Press.

Williamson (Oliver E.), 1986, Economic Organisation. Firms, markets and policy control, New York, New York University Press, p. 142-164.

Williamson (O. E.), 1979, «Transaction Cost Economics: the Governance of Contractual Relations », Journal of Law and Economics, 22, p. 233-261.

Williamson (O. E.), 1985, The Economic Institutions of Capitalism: Firms, Markets, Relational Contracting, New York, Free Press.

Wilson (John F.), Popp (Andrew) (dir.), 2003, Industrial Clusters and Regional Business Networks in England, 1750-1970, Aldershot, Ashgate. 